THERAPY

\section{Expanding the horizons of PARP inhibitors}

\begin{abstract}
Aberrant vascularity in tumours results in hypoxic tumour regions that are distant from blood vessels, as oxygen is poorly delivered to these areas. As drugs are also not efficiently delivered in these circumstances, intratumoral hypoxia is associated with treatment resistance. Rob Bristow and Ester Hammond have come up with a possible solution to selectively target resistant hypoxic tumour cells.

It is known that the ability to repair DNA damage by the homologous recombination pathway is impaired in hypoxic cells and that inhibition of poly(ADP-ribose) polymerase 1 (PARP1) specifically kills cancer cells that have defects in
\end{abstract}

Rob Bristow and Ester Hammond have come up with a possible solution to selectively target resistant hypoxic tumour cells. this pathway - such as mutations in $B R C A 1$ or BRCA2 - through a synthetic lethal effect. These two factors prompted the authors to speculate that homologous recombinationdefective hypoxic cells might also be sensitized to PARP inhibitors. To assess this, the authors first evaluated the effects of hypoxia on the homologous recombination pathway in a panel of human cancer cell lines and xenografts and observed that the homologous recombination protein RAD51 was decreased under hypoxia both in vitro and in vivo. This effect was independent of whether the cells expressed PARP or hypoxia inducible factor $1 \alpha(H I F 1 \alpha)$. The authors then investigated the effects of hypoxia

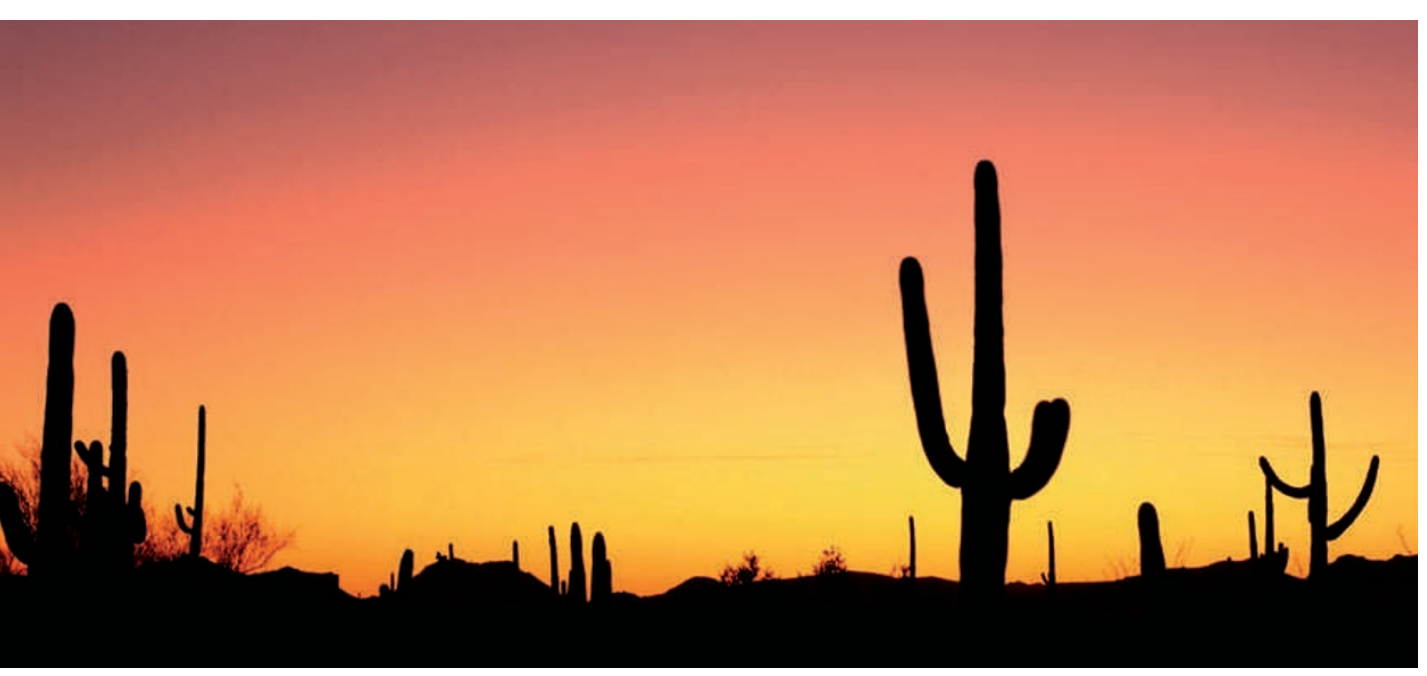

on PARP inhibition by analysing the proliferation rate of PARP1-deficient mouse embryonic fibroblasts (MEFs) in hypoxic conditions and found that they proliferated more slowly than under normal levels of oxygen. Accordingly, the survival of tumour cells was dramatically decreased after treatment with PARP inhibitors under acute or prolonged hypoxia, and cell survival was partially recovered when RAD51 was overexpressed. The authors concluded that this result could be explained by the fact that PARP inhibition leads to an accumulation of DNA damage in hypoxic cells, and that this damage can be repaired when the homologous recombination pathway is re-established.

To determine whether hypoxic cells can be selectively killed by PARP inhibition in vivo, the authors treated nude mice bearing human colon cancer xenografts with PARP inhibitors and then irradiated the tumours after the last dose of the treatment. Irradiated tumours that had been pretreated with PARP inhibitors had lower survival than control xenografts that were treated with just vehicle; notably, the combination of irradiation and PARP inhibition did not kill non-tumour cells. These results therefore expand the possibilities of PARP inhibitors in the clinic by specifically targeting hypoxic tumour cells that are resistant to radiotherapy or chemotherapy.

Teresa Villanueva

ORIGINAL RESEARCH PAPER Chan, N. et al. Contextual synthetic lethality of cancer cell kill based on the tumour microenvironment. Cancer Res. 70, 8045-8054 (2010) 